\title{
Krebskranke mit Depression sterben früher
}

\author{
Eine Depression ist bei Patienten \\ mit Krebs häufig (13-40\%). Voran- \\ gegangene Arbeiten wiesen darauf \\ hin, dass eine Depression mit einer \\ erhöhten Sterblichkeit assoziiert sein \\ könnte. Das Ziel der vorliegenden \\ Metaanalyse war eine Reevaluation \\ des Zusammenhangs in einer großen \\ Stichprobe und eine weitere Analyse \\ des Einflusses verschiedener Mode- \\ ratorvariablen wie der Schwere der \\ Erkrankung.
}

— Insgesamt 76 Studien wurden in die vorliegende Analyse eingeschlossen ( $\mathrm{n}=$ 176863 Patienten). Die Metaanalyse nutzte Random-Effects-Modelle. Die Assoziation zwischen einer Depression und der Mortalität wurde als relatives Risiko ausgedrückt (Risiko für Sterblichkeit in der Gruppe mit Depression vs. ohne Depression).

Die in die Metaanalyse eingeschlossenen Patienten (72\% Frauen) waren im Mittel 64,5 Jahre alt $(S D=11,6)$. Die meisten Studien untersuchten den Einfluss depressiver Episoden oder depressiver Symptome nach Stellung der Diagnose „Krebs“. Die Beobachtungszeiträume variierten zwischen weniger als zwei und mehr als sechs Jahren. Über 90\% der Studien berichteten ein relatives Risiko $>1,0$ sowohl für depressive Episoden (kategoriale Diagnosestellung) als auch für depressive Symptome (dimensionale Erfassung). Dies weist darauf hin, dass eine Depression mit einer erhöhten Sterblichkeit assoziiert ist (gemitteltes relatives Risiko 1,19, 95\%-Konfidenzintervall [KI] 1,13-1,25, Z =6,48, $\mathrm{p}<0,001)$. Die Stärke des Zusammenhangs war signifikant vom Alter abhängig (ausgeprägter bei älteren Patienten), nicht jedoch vom Geschlecht oder von der Art oder dem Stadium des Tumors.

Depression ist mit einer erhöhten Sterblichkeit bei Patienten mit Krebserkrankungen assoziiert. Weitere Untersu-

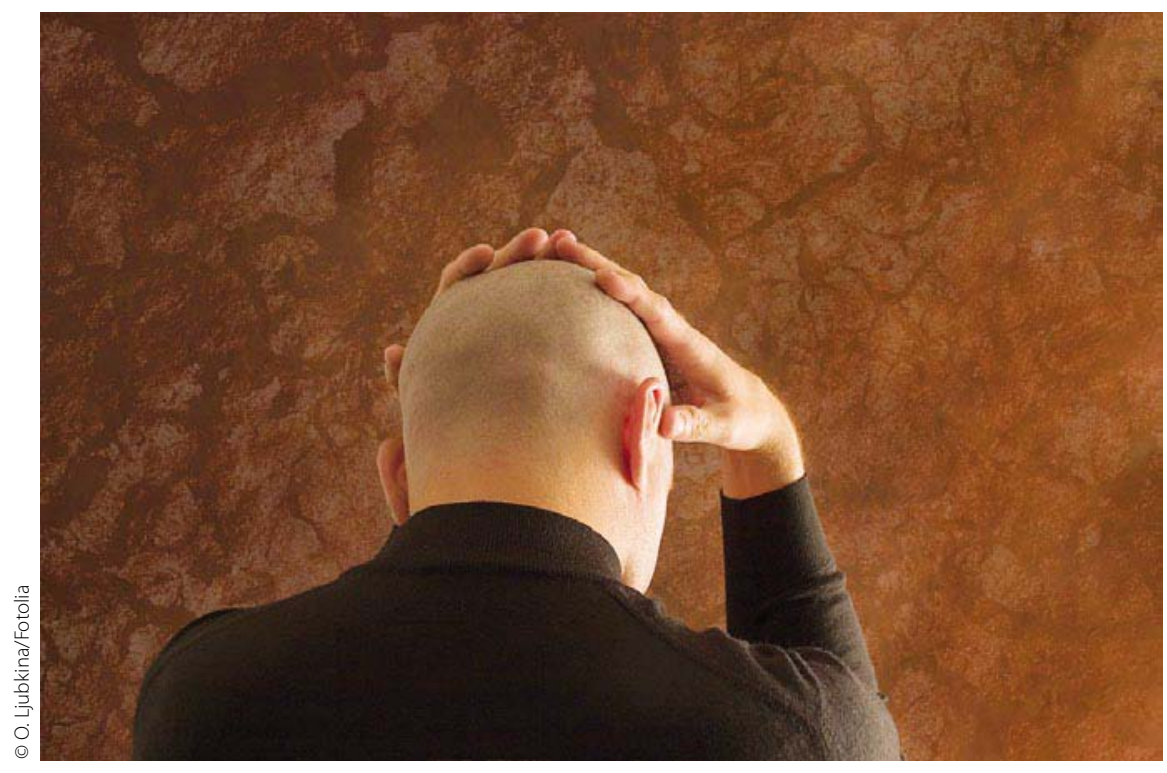

Können Antidepressiva das Leben von Krebspatienten verlängern?

chungen sind notwendig, um zu prüfen, ob eine antidepressive Behandlung die Überlebensrate von Patienten mit onkologischen Erkrankungen verbessert.

\section{Kommentar}

Die hier vorliegende Metaanalyse zeigt eine robuste Assoziation zwischen depressiven Symptomen und einer erhöhten Mortalität bei Patienten mit Krebserkrankungen (Evidenzstufe l). Patienten mit depressiven Symptomen wiesen eine um rund $20 \%$ erhöhte Sterblichkeit gegenüber Patienten ohne relevante depressive Symptome auf. Dieser Zusammenhang war nicht durch die Schwere der Erkrankung erklärbar. Verschiedene Fragen konnten in der hier vorliegenden Untersuchung nur unzureichend beantwortet werden. So bleiben die genaueren Gründe des Zusammenhangs aktuell offen. Denkbar sind psychosoziale Faktoren wie die Inanspruchnahme des Gesundheitssystems oder die Compliance bezüglich der Behandlung, aber auch biolo- gische Faktoren wie immunologische Veränderungen bleiben zu diskutieren. Aufgrund der methodischen Vielfalt der Studien war eine genaue Untersuchung des Einflusses der Tumorart, der Symptomkonstellation der Depression und der Behandlung nicht möglich. Unabhängig von diesen Fragen ist zu empfehlen, Patienten mit Krebserkrankungen sorgfältig bezüglich depressiver Symptome zu screenen. Antidepressive Interventionen haben in vorangegangenen Studien die Depressivität und die Lebensqualität bei Patienten mit Krebs verbessert. Es liegt nahe, dass diese Interventionen auch die Überlebenszeit verlängern könnten. Diesbezügliche Studien stehen jedoch aktuell aus.

Christoph Nissen, Freiburg i. Br. -

- M. Pinquart, P. R. Duberstein Depression and cancer mortality: a meta-analysis. Psychol. Med. 20 (2010) 1-14 